LA-UR-01-0655

Approved for public release; distribution is unlimited.

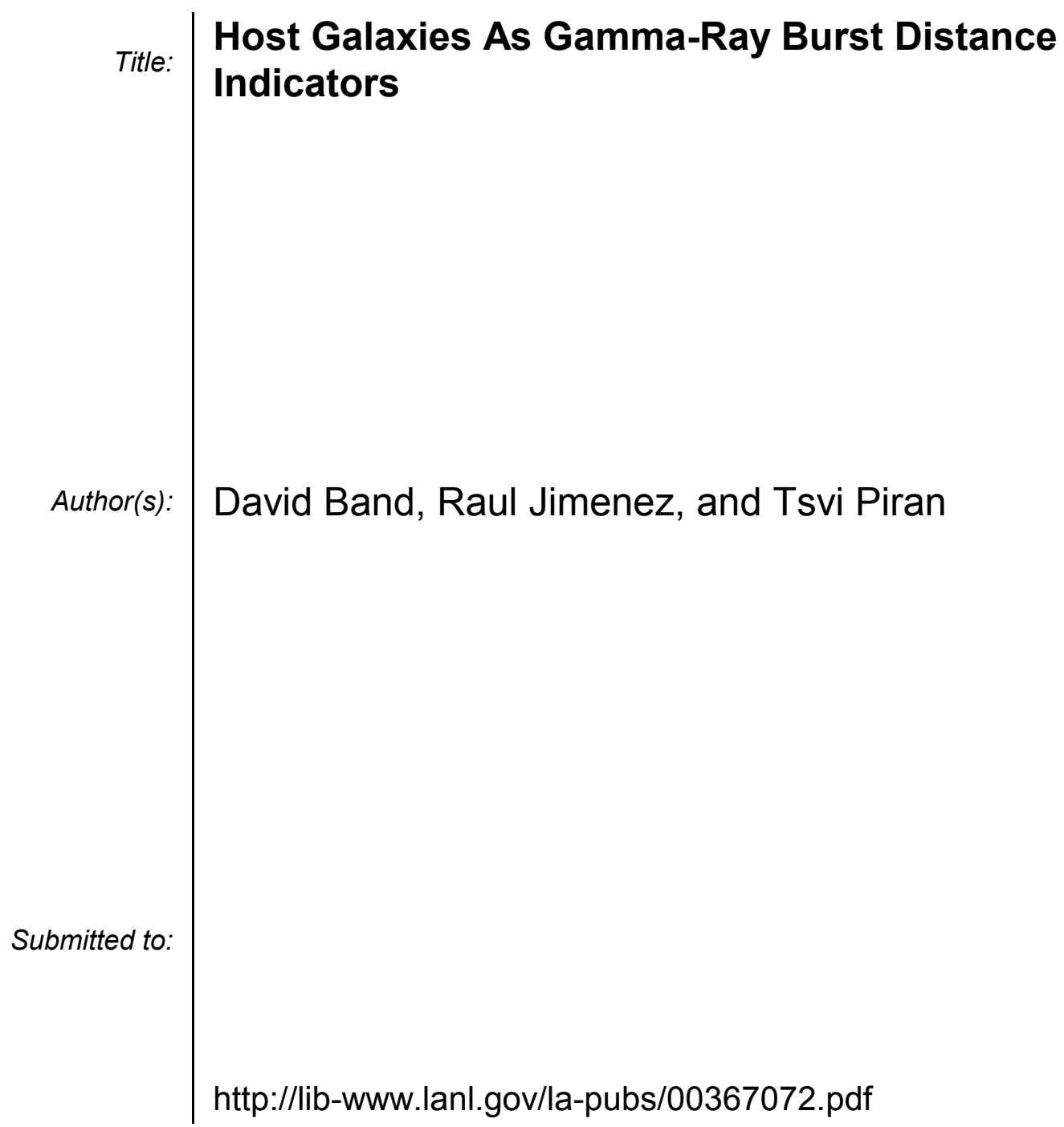

Los Alamos National Laboratory, an affirmative action/equal opportunity employer, is operated by the University of California for the U.S. Department of Energy under contract W-7405-ENG-36. By acceptance of this article, the publisher recognizes that the U.S. Government retains a nonexclusive, royaltyfree license to publish or reproduce the published form of this contribution, or to allow others to do so, for U.S. Government purposes. Los Alamos National Laboratory requests that the publisher identify this article as work performed under the auspices of the U.S. Department of Energy. Los Alamos National Laboratory strongly supports academic freedom and a researcher's right to publish; as an institution, however, the Laboratory does not endorse the viewpoint of a publication or guarantee its technical correctness. 


\title{
Host Galaxies As Gamma-Ray Burst Distance Indicators
}

\author{
David Band ${ }^{1}$, Raul Jimenez ${ }^{2}$, and Tsvi Piran ${ }^{3}$ \\ 1 X-2, Los Alamos National Laboratory, Los Alamos, NM 87505, USA \\ 2 Dept. of Physics and Astronomy, Rutgers University, Piscataway, NJ 08854, USA \\ 3 Hebrew University, Jerusalem, Israel
}

\begin{abstract}
We calculate the distributions of the total burst energy, the peak luminosity and the X-ray afterglow energy using burst observations and distances to the associated host galaxies. To expand the sample, we include redshift estimates for host galaxies without spectroscopic redshifts. The methodology requires a model of the host galaxy population; we find that in the best model the burst rate is proportional to the host galaxy luminosity at the time of the burst.
\end{abstract}

\section{Introduction}

The first few bursts with afterglows, host galaxies and spectroscopic redshifts demonstrated that most, if not all, classical bursts occur at cosmological distances. As the sample of such bursts grows, we can address fundamental characteristics of the burst population. Here we calculate the distributions of the total gamma-ray energy emitted, the peak luminosity, and the energy of the Xray afterglow. The average values of these quantities set fundamental physical constraints on burst models, while the widths of the distributions indicate the variability these models must permit.

To estimate the distribution of these different intrinsic intensities requires both the apparent intensity of the burst and the distance to the burst. We fit spectra to BATSE data, where available, to calculate the energy fluence and the peak luminosity. The distance is obtained from the spectroscopic redshift, if observed, or from a redshift probability distribution derived from the host galaxy brightness. We include bursts without spectroscopic redshifts out of a desire to use all available data. The number of bursts with spectroscopic redshifts has increased since we began this project, and the inclusion of bursts without such redshifts is not as compelling, although the methodology will again be useful if the determination of spectroscopic redshifts does not keep up with the discovery of host galaxies. The redshift probability distribution requires a model of the host galaxy population, which we choose using a sample of bursts with both a spectroscopic redshift and a host galaxy brightness. This host galaxy population model is intrinsically interesting since it depends on the type of progenitor.

\section{Methodology}

We present the methodology for determining the distribution of the gammaray energy, but the approach is easily generalized to the distributions of the 
peak gamma-ray luminosity and the X-ray afterglow energy. We assume that the gamma-ray energy is characterized by a lognormal burst distribution. We convert the energy to an observed fluence using the redshift and the cosmological model with $H_{0}=65 \mathrm{~km} \mathrm{~s}^{-1} \mathrm{Mpc}^{-1}, \Omega_{M}=0.3$ and $\Omega_{\Lambda}=0.7$. As discussed above, we use the spectroscopic redshift, if available; otherwise we use an estimated redshift probability distribution $p_{\mathrm{B}}\left(z \mid D_{0}\right)$ based on the host galaxy's $\mathrm{R}$ magnitude, as described below. The result is a probability distribution for the fluence for each burst parameterized by the average burst energy and the logarithmic width of the energy distribution.

The likelihood function is the product of the probability of observing each fluence. This probability is the probability distribution for the burst fluence above the fluence threshold, the minimum fluence for which the burst would be included in our sample. Using the fluence threshold mitigates the selection effect where at high redshift only intrinsically bright bursts can be detected. We then maximize this likelihood by varying the parameters of the lognormal distribution - the average energy and the logarithmic width of the distribution; confidence ranges are also determined.

Our sample consists of bursts with spectroscopic redshifts or host galaxy detections which were observed by BATSE between 1997 and CGRO's untimely demise in June, 2000. We fit BATSE spectra with the four parameter "GRB" function [1]. The fit to the spectrum accumulated over an entire burst was integrated over the $20-2000 \mathrm{keV}$ energy range and the burst duration to give that burst's bolometric fluence, while the spectrum accumulated over the shortest available period which included the burst's peak count rate was integrated over $50-300 \mathrm{keV}$ in the burst's frame to provide the peak luminosity. We find that fluences derived by integrating over spectral fits are a factor of $\sim 2$ smaller than the values in the online BATSE catalog [2], but given the large range of fluence values, this discrepancy is insignificant. In many bursts most of the energy is above $300 \mathrm{keV}$ where the fits are uncertain.

The X-ray afterglow was first extrapolated back to $100 \mathrm{~s}$ after the burst using the observed X-ray flux and power-law temporal decay. The X-ray afterglow was then integrated over time and frequency to provide the total X-ray afterglow energy. Since the temporal decay is usually close to $t^{-1}$, the calculated energy depends only logarithmically on the uncertain beginning of the afterglow.

For the bursts without a spectroscopic redshift we need $p_{\mathrm{B}}(z \mid R)$, the probability distribution that a host galaxy with magnitude $\mathrm{R}$ is at redshift $\mathrm{z}$. Empirically, the Hubble Deep Field (HDF) provides $p_{\mathrm{HDF}}(z \mid R)$, the probability that a galaxy with magnitude $\mathrm{R}$ is at redshift $\mathrm{z}$. However, this counts each galaxy equally - a nearby dwarf is equal to a distant luminous galaxy. Thus, we must weight $p_{\mathrm{HDF}}$ by the probability that the galaxy is a host, which requires a model of the host galaxy population. We consider four models. In the first, bursts are equally likely to occur in any galaxy and thus $p_{\mathrm{HDF}}$ is unweighted. In the second, the burst rate is proportional to the galaxy mass; $p_{\mathrm{HDF}}$ is weighted by the luminosity with $\mathrm{k}$ - and e-corrections. In the third, the burst rate is proportional to the galaxy luminosity at the time of the burst; $p_{\mathrm{HDF}}$ is weighted by the luminos- 
ity with only k-corrections. Finally, in the fourth, the burst rate is proportional to the galaxy mass times the cosmic star formation rate; the distribution from model 2 is multiplied by this star formation rate.

We choose the host galaxy model which best fits the set of 10 bursts with both spectroscopic redshifts and host galaxy $\mathrm{R}$ magnitudes. For each model we form a likelihood which is the product of $p_{\mathrm{B}}$ for each burst in this dataset; each model has a different $p_{\mathrm{B}}$. Technically $p_{\mathrm{B}}$ should be modified to account for the redshift ranges in which the observing telescope would have been unable to determine the redshift. However, accounting for the capabilities of all the different telescopes is beyond the scope of this work. We find the following likelihoods for the different models: model $1-4 \times 10^{-3}$; model $2-2 \times 10^{-4}$; model $3-4 \times 10^{-2}$; and model 4 $-10^{-4}$. Thus for all our calculations we use model 3 , where the burst rate is proportional to the galaxy's luminosity at the time of the burst.

\section{Results and Discussion}

By applying the methodology described above to a sample of 9 bursts with spectroscopic redshifts and 3 bursts with only host galaxy magnitudes we find that the distribution for the total energy is characterized by $\left\langle E_{\gamma}\right\rangle=1.6_{-1.2}^{+1.7} \times$ $10^{53} \mathrm{erg}$ with a logarithmic width of $\sigma_{\gamma}=1.7_{-0.4}^{+0.8}$. We emphasize that this is the total energy if the burst radiates isotropically. We use natural logarithms in defining the width. The uncertainties are $2 \sigma$. Using the same dataset we find $\left\langle E_{L}\right\rangle=4.6_{-2.5}^{+5.4} \times 10^{51} \mathrm{erg} \mathrm{s}^{-1}$ and $\sigma_{L}=1.4_{-0.35}^{+0.7}$ for the peak gammaray luminosity. For the afterglow X-ray energy we have $\left\langle E_{X}\right\rangle=4.5_{-2.8}^{+6.7} \times 10^{51}$ erg and $\sigma_{X}=1.6_{-0.55}^{+0.85}$; this was calculated using 6 bursts with redshifts and 1 with only a host $\mathrm{R}$ magnitude. The total energy radiated is enormous, even with beaming factors which may reduce the actual energy release by one or two orders of magnitude. Of course, the actual energy release must be even greater because of the imperfect transfer of energy into radiating particles and then into emission. The large energies required severely constrain the physical models.

This methodology can be used wherever burst distances and energies are required. For example, a burst sample with known distances is required to calibrate proposed correlations between the burst energy and the frequency-dependent lags in pulses [3] or lightcurve variability [4].

\section{References}

1. D. Band, et al.: Astrophys. J. 413, 281 (1993)

2. BATSE GRB team: http://www.batse.msfc.nasa.gov/batse/grb/catalog/current/ (2001)

3. E. Fenimore, E. Ramirez-Ruiz: Astrophys. J., submitted, astro-ph/0004176 (2001)

4. J. Norris, G. Marani, and J. Bonnel: Astrophys. J. 534, 248 (2000) 